\title{
Predicted Area Under the Urinary Excretion Rate Curve Percent Extrapolation
}

National Cancer Institute

\section{Source}

National Cancer Institute. Predicted Area Under the Urinary Excretion Rate Curve Percent Extrapolation. NCl Thesaurus. Code C85792.

The percentage of the area under the urine excretion rate curve (AURC) extrapolated to infinity predicted from Tlast to infinity. 